\title{
Congenital anomalies of reproductive system among infertile women attending Dr. Khawer Center for Infertility and IVF in Maternity Teaching Hospital in Erbil city: A retrospective study
}

\begin{tabular}{rr}
\hline Rana Muhammed Taha Al-Attar ${ }^{\text {* }}$ & Yasin Kareem Amin ${ }^{2}$ \\
\hline Abstract &
\end{tabular}

Background and objective: Congenital anomalies of the female genital tract is a known cause for infertility and recurrent pregnancy losses. This study aimed to evaluate the pattern and prevalence of each type of congenital malformation of the reproductive tract among infertile women attending the IVF center in the Maternity Teaching Hospital in Erbil city.

Methods: A retrospective study was conducted in Center of Dr. Khawer for Infertility and IVF in the Maternity Teaching Hospital in Erbil city. Data of five years from the $1^{\text {st }}$ January 2010 to the $1^{\text {st }}$ January 2015 were collected from patient files, such as age, sex, residence, type of infertility, type of anomaly, duration of infertility, Karyotype, Intervention, Success rate of intervention, cycle, parity, history of abortions, history of preterm deliveries, and history of ectopic pregnancy.

Results: The prevalence of congenital reproductive system anomaly was $4.1 \%$ among infertile women. The sub-septate uterus was the most common type $(23.24 \%)$ anomalies. No significant association was found between the types of the anomaly and the type of infertility. The genetic factor was found to has a significant role in developing such a condition. Success rate after management significantly raised to $61.1 \%$ with sub-septate uterus $(91.3 \%)$ bicornuate/ partial $(85.7 \%)$, and septate/ complete $(72.2 \%)$.

Conclusion: One of the causative factors of female infertility is congenital anomaly. Most types have a good prognosis in achieving pregnancy after proper management.

Keywords: Congenital; Sub-septate; Anomaly; Types; Reproductive tract.

\section{Introduction}

Congenital anomaly of the reproductive system is regarded as one of the leading causes of adverse reproductive outcomes, physical and psychosocial problems. ${ }^{1,2}$ They occur during intrauterine life during the embryonic period which lasts from $3^{\text {rd }}$ to $8^{\text {th }}$ weeks of gestation resulting from abnormal mesonephric or paramesonephric ductal development unilaterally or bilaterally. ${ }^{3-5}$ Due to various reasons like chromosomal abnormalities, environmental factors, multifactorial or idiopathic. $^{6-8}$ They have different impacts on human reproductive function differs according to the type of the anomaly and the extent of anatomical distortion, affected individuals may have either entirely normal fertility and sexual life, or it can lead to serious sexual and fertility problems like amenorrhea, severe dysmenorrhea, miscarriage, preterm delivery, and most importantly infertility. ${ }^{1,10-12}$ Although the exact prevalence of female genital tract anomalies is difficult to be known, as many cases are asymptomatic and not detected. ${ }^{13}$ It is estimated that the prevalence of such anomaly in the general population to be around $4-7 \% .{ }^{14}$ In a study conducted in 2011, the prevalence of uterine anomalies was $5.5 \%$ among unselected general population and $8 \%$ among infertile women, while it was $13.3 \%$ among those with history of miscarriage

${ }_{1}^{1}$ Erbil Directorate of Health, Erbil, I raq.

2 Medical Research Center, Hawler Medical University, Erbil, I raq.

* Correspondence: aynuralattar@gmail.com 
and $24.5 \%$ among infertile women with a history of miscarriage. ${ }^{18}$ In a recent study from southwest Nigeriain 2015, it was found that the sub-septate uterus was the most common type among infertile women. ${ }^{15}$ While Arcuate uterus was found to be the most common anomaly type in the unselected population ${ }^{3,9,18} \mathrm{Up}$ to researchers' knowledge, this is the first study for assessment of congenital anomalies of the female reproductive tract as an etiological factor of infertility in the infertility center of Maternity Teaching Hospital in Erbil city. This study aimed to evaluate the prevalence of congenital anomalies causing women infertility, the distribution of anomaly types, type of infertility they cause, karyotype abnormalities among them, and success rate for achieving pregnancy in each type after an intervention.

\section{Methods \\ Study design}

This study was carried out in the Center of Dr. Khawer for Infertility and IVF in the Maternity Teaching Hospital in Erbil city, Kurdistan region, Iraq. Data were collected from medical records of 2337 files in which 96 cases aged between 18-45 years with diagnosed female reproductive system anomalies were detected. The cases were diagnosed by history, clinical examination, imaging, and investigations. Pelvic or vaginal two-dimensional ultrasonography, 3 -D ultrasonography, hysterosalpingography and hysteroscopy were mostly used diagnostic tools for that reason. The collection process lasted for four months, data of five years were collected, from the $1^{\text {st }}$ January 2010 to the $1^{\text {st }}$ January 2015. This study was approved by the Research Ethics Committee at the College of Medicine, Hawler Medical University

Inclusion and exclusion criteria

In this study, all cases from inside Erbil city or surrounding nearby places were included as the study investigated the congenital anomaly in Erbil city. All cases who had all required data records in their files and who are managed completely from the diagnosis to the end after treatment. Cases in which the congenital anomaly was the exact cause of infertility without coexisting other causes. Excluded cases were cases who referred from outside Erbil city, cases with missed data due to improper recording, lack of patient's compliance for follow up or completing lines of management, cases associated with anatomical problem such as polyp as the exact cause of infertility obscured, cases in which both partners have problems simultaneously like presence of arcuate uterus in female while the husband presented azoospermia, cases not belonging to any classes selected in AFS classification system. Data such as age, residence, type of infertility, type of anomaly, duration of infertility, parity, history of abortions, karyotype, intervention, and success rate of intervention were collected. Congenital anomalies in this study were grouped retrospectively according to the American classification system AFS, ${ }^{1,16}$ which classified anomalies to seven main classes and their subclasses. Class-I-Hypoplasia/ agenesis has five subclasses of vaginal, cervical, uterine, tubal, and combined anomalies. Class-II unicornuate uterus is divided into four subclasses unicornuate with a communicating rudimentary horn, unicornuate with a non-communicating rudimentary horn, unicornuate with a rudimentary horn containing no uterine cavity and unicornuate without a horn. Class-III didelphys uterus (double uterus). Class-IV bicornuateuterus which is subdivided into two classes as bicornuate uterus with complete division down to the internal os and bicornuate uterus with the partial division. Class- $V$ septate uterus in which anomalies are subdivided into two classes septate uterus with complete septum down to the external os and septate uterus with partial septum not reaching the os. Class $\mathrm{VI}$-arcuate uterus and class-VII drug induced (DES) $T$ shaped uterus during intrauterine life. A group of gonadal 
dysgenesis added to the categories of the classification system because the system classified only Mullerian anomalies and mostly focused on uterus, while other parts like ovaries and sex determination are not included. Our study included anomalies throughout the reproductive system due to the availability of many cases of gonadal dysgenesis cases causing infertility. The type of infertility was divided as primary and secondary infertility. Chromosomal abnormalities as a causative factor for the anomaly were categorized as normal or abnormal karyotype, according to the karyotype study results which had been performed for the patients. The success rate of the intervention was subdivided into either pregnancy achieved or not achieved, while age, parity, and history of abortion entered as numerical data. Data were analyzed using the statistical package for the social sciences (version 22). Means and standard deviations were used to summarize the numerical variables. Frequencies and percentages were used to present and summarize the categorical variables. The Chi-square test of association was used to compare proportions. Fisher's exact test was used when the expected count of more than $20 \%$ of the cells of the table was less than 5. A $P$ value of $\leq 0.05$ was considered statistically significant.

\section{Results}

Out of 2337 infertile women, $96(4.1 \%)$ had congenital anomalies in their reproductive system. The mean age ( \pm SD) was $30.46 \pm$ 6.39 years, ranging from $18-45$ years. The median age was 29.5 years. Figure 1 shows that septate uterus is the most common type among other types of anomaly $45.47 \%$. Around $23 \%$ of the sample had septate/partial (sub-septate) uterus, $22.23 \%$ had septate/complete uterus, $12 \%$ had uterus didelphys, $8 \%$ had bicornuate/partial uterus, and $7 \%$ had arcuate uterus. Other anomalies are presented in Figure 1 and were rare, while no cases of the vaginal and $t$-shaped uterus could be found.

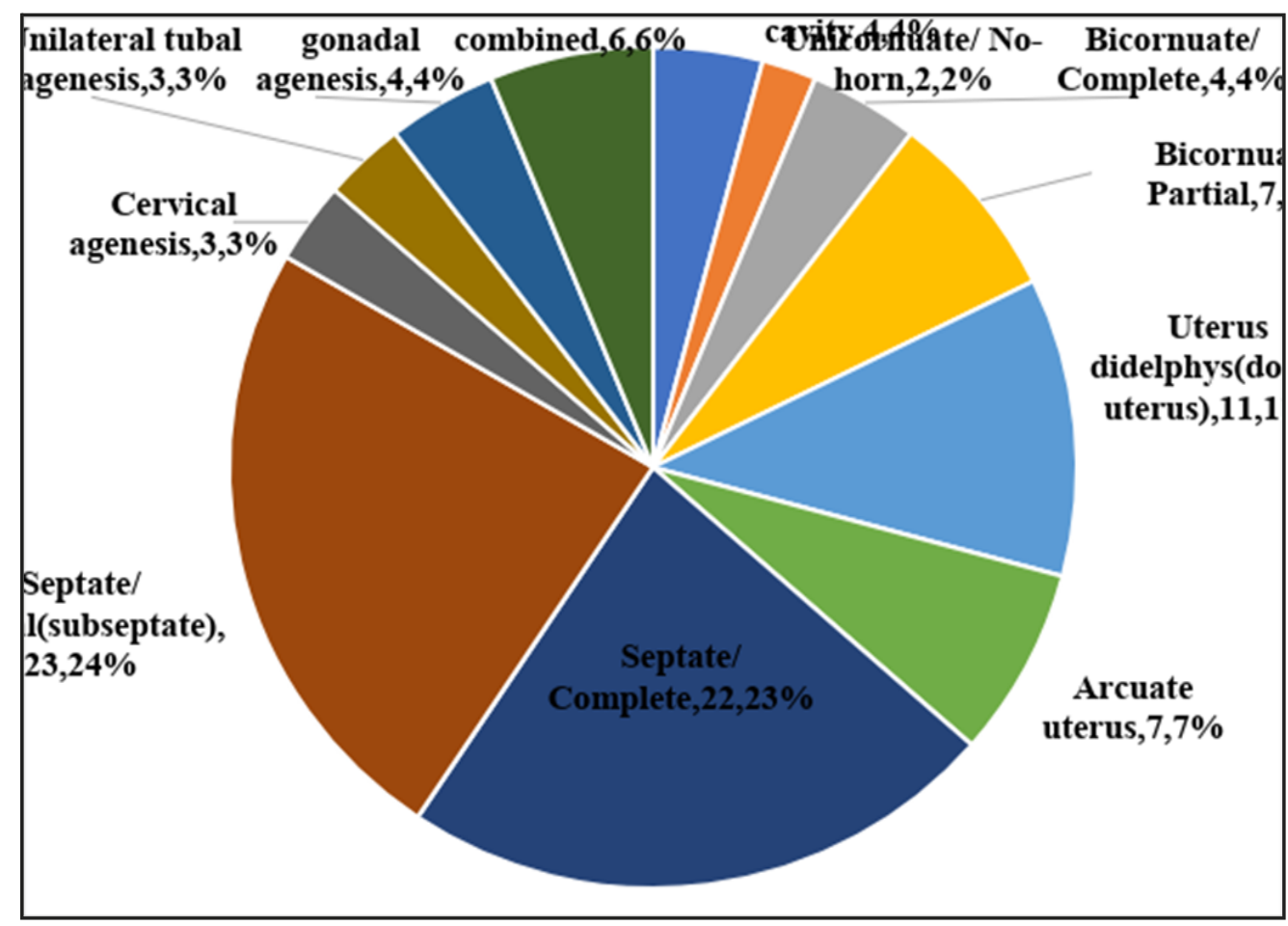

Figure 1: Distribution of female sample by type of anomaly. 
Table 1 shows that fifty-one women $(53.1 \%)$ had primary infertility, and the rest $(46.9 \%)$ had secondary infertility. All of those with unilateral tubal agenesis and those with gonadal agenesis had primary infertility. No significant association was detected between the type of infertility and the type of anomaly $(P=0.395)$. Eleven women out of 96 cases $(11.5 \%)$ had karyotype abnormality as presented in Table 2. Women with the following anomalies had karyotype abnormality: bicornuate/ complete uterus (50\%), gonadal agenesis (100\%), and combined $(83.3 \%)$. A significant association was detected between karyotype abnormalities with the type of anomalies $(P<0.001)$.

Table 1: The relationship between type of anomalies and type of infertilities.

\begin{tabular}{lcccccc}
\hline & \multicolumn{3}{c}{ Type of infertility } & \multicolumn{2}{c}{ Total } \\
Type of anomaly & \multicolumn{2}{c}{ Primary } & \multicolumn{2}{c}{ Secondary } & \multicolumn{2}{c}{$\%$} \\
\hline Unicornuate/ No-cavity & No. & $\%$ & No. & $\%$ & No. & $(100.0)$ \\
Unicornuate/ No-horn & 2 & $(50.0)$ & 2 & $(50.0)$ & 4 & $(100.0)$ \\
Bicornuate/ Complete & 1 & $(50.0)$ & 1 & $(50.0)$ & 2 & $(100.0)$ \\
Bicornuate/ Partial & 3 & $(75.0)$ & 1 & $(25.0)$ & 4 & $(100.0)$ \\
Uterus didelphys(double uterus) & 3 & $(42.9)$ & 4 & $(57.1)$ & 7 & $(100.0)$ \\
Arcuate uterus & 6 & $(54.5)$ & 5 & $(45.5)$ & 11 & $(100.0)$ \\
Septate/ Complete & 3 & $(42.9)$ & 4 & $(57.1)$ & 7 & $(100.0)$ \\
Septate/ partial (subseptate) & 9 & $(40.9)$ & 13 & $(59.1)$ & 22 & $(100.0)$ \\
Cervical agenesis & 11 & $(47.8)$ & 12 & $(52.2)$ & 23 & $(100.0)$ \\
Unilateral tubal agenesis & 1 & $(33.3)$ & 2 & $(66.7)$ & 3 & $(100.0)$ \\
Gonadal agenesis & 3 & $(100.0)$ & 0 & $(0.0)$ & 3 & $(100.0)$ \\
Combined & 4 & $(100.0)$ & 0 & $(0.0)$ & 4 & $(100.0)$ \\
Total & 5 & $(83.3)$ & 1 & $(16.7)$ & 6 & $(100.0)$ \\
\hline P & 51 & $(53.1)$ & 45 & $(46.9)$ & 96 &
\end{tabular}

$P=0.395$ (By Fisher's exact test).

Table 2: The relationship between Karyotype abnormality and type of anomaly.

\begin{tabular}{lcccccc}
\hline & \multicolumn{2}{c}{ Karyotype } & & \multicolumn{2}{c}{ Total } \\
Type of anomaly & No. & $(\%)$ & No. & (\%) & No. & (\%) \\
\hline Unicornuate/ No-cavity & 4 & $(100.0)$ & 0 & $(0.0)$ & 4 & $(100.0)$ \\
Unicornuate/ No-horn & 2 & $(100.0)$ & 0 & $(0.0)$ & 2 & $(100.0)$ \\
Bicornuate/ Complete & 2 & $(50.0)$ & 2 & $(50.0)$ & 4 & $(100.0)$ \\
Bicornuate/ Partial & 7 & $(100.0)$ & 0 & $(0.0)$ & 7 & $(100.0)$ \\
Uterus didelphys(double uterus) & 11 & $(100.0)$ & 0 & $(0.0)$ & 11 & $(100.0)$ \\
Arcuate uterus & 7 & $(100.0)$ & 0 & $(0.0)$ & 7 & $(100.0)$ \\
Septate/ Complete & 22 & $(100.0)$ & 0 & $(0.0)$ & 22 & $(100.0)$ \\
Septate/ Partial(subseptate) & 23 & $(100.0)$ & 0 & $(0.0)$ & 23 & $(100.0)$ \\
Cervical agenesis & 3 & $(100.0)$ & 0 & $(0.0)$ & 3 & $(100.0)$ \\
Unilateral tubal agenesis & 3 & $(100.0)$ & 0 & $(0.0)$ & 3 & $(100.0)$ \\
Gonadal agenesis & 0 & $(0.0)$ & 4 & $(100.0)$ & 4 & $(100.0)$ \\
Combined & 1 & $(16.7)$ & 5 & $(83.3)$ & 6 & $(100.0)$ \\
Total & 85 & $(88.5)$ & 11 & $(11.5)$ & 96 & $(100.0)$ \\
\hline$P<0.001$ (by Fisher's exact test). & & & & & & \\
\hline
\end{tabular}


The success rate (pregnancy achieved) after the intervention was $61.1 \%$, as presented in Table 3 . The highest pregnancy rate was among women with septate/ partial uterus $(91.3 \%)$, so it has the best prognosis. They followed by women with bicornuate/partial uterus $(85.7 \%)$, and women with septate/complete uterus $(72.2 \%)$, while none of the women with unicornuate uterus, bicornuate complete uterus, gonadal agenesis, and the combined conceived. There was a significant association between the type of anomaly and achieving pregnancy after management $(P<0.001)$.

\section{Discussion}

Our study evaluated the prevalence and pattern of congenital reproductive system anomalies among a mostly homogeneous population of infertile women who attended the Center of Dr. Khawer for Infertility and IVF in Maternity Teaching Hospital in Erbil city. Anomalies of the reproductive tract are obscured and remain undetected until puberty or childbearing age on most occasions, as they usually not interfere adversely with normal physiological functions until that time. This study raises awareness of congenital genital malformations while assessing causes of infertility also aids in the establishment of better programs for the diagnosis and management of such problems in Erbil city. The prevalence of the female reproductive system among infertile women was $4.1 \%$, which is near to the result of a hospital-based study conducted in Mosul city $(5.68 \%) .{ }^{17}$ However, it disagrees with a study reported by Chan in Nottingham in which $8 \%$ of women infertility caused by congenital malformation. ${ }^{18}$ The difference between countries is possibly related to differences in environmental factors, the role of genetic factor, lifestyle, nutrition, and racial differences in the distribution

Table 3: Success of intervention by type of anomaly.

\begin{tabular}{lcccccc}
\hline & \multicolumn{3}{c}{ Achieved } & \multicolumn{2}{c}{ Not achieved } & \multicolumn{2}{c}{ Total } \\
Type of anomaly & No. & $(\%)$ & No. & $(\%)$ & No. & $(\%)$ \\
\hline Unicornuate/ No-cavity & 0 & $(0.0)$ & 4 & $(100.0)$ & 4 & $(100.0)$ \\
Unicornuate/ No-horn & 0 & $(0.0)$ & 2 & $(100.0)$ & 2 & $(100.0)$ \\
Bicornuate/ Complete & 0 & $(0.0)$ & 4 & $(100.0)$ & 4 & $(100.0)$ \\
Bicornuate/ Partial & 6 & $(85.7)$ & 1 & $(14.3)$ & 7 & $(100.0)$ \\
Uterus didelphys(double uterus) & 6 & $(54.5)$ & 5 & $(45.5)$ & 11 & $(100.0)$ \\
Arcuate uterus & 3 & $(42.9)$ & 4 & $(57.1)$ & 7 & $(100.0)$ \\
Septate/ Complete & 16 & $(72.2)$ & 6 & $(27.3)$ & 22 & $(100.0)$ \\
Septate/ Partial(subseptate) & 21 & $(91.3)$ & 2 & $(8.7)$ & 23 & $(100.0)$ \\
Cervical agenesis & 1 & $(33.3)$ & 2 & $(66.7)$ & 3 & $(100.0)$ \\
Unilateral tubal agenesis & 2 & $(66.7)$ & 1 & $(33.3)$ & 3 & $(100.0)$ \\
Gonadal agenesis & 0 & $(0.0$ & 1 & $(100.0)$ & 1 & $(100.0)$ \\
Combined & 0 & $(0.0)$ & 3 & $(100.0)$ & 3 & $(100.0)$ \\
Total & 55 & $(61.1)$ & 35 & $(38.9)$ & 90 & $(100.0)$ \\
\hline P<0.001)
\end{tabular}

$P<0.001$ (by Fisher's exact test). 
of the anomaly, or maybe due to better diagnostic measures there. ${ }^{1}$ Septate uterus, which is a canalization defect, was found to be the most common type of reproductive tract malformation among infertile women in this study (sub-septate $23.24 \%$ and complete septum $22.23 \%$ ). This is because the strong muscular septum predisposes to failure of embryo implantation and decreases intrauterine space leading to early pregnancy loss due to interfering with fetal growth and physiological function of the placenta by diminishing its blood supply. A study conducted in the UK supports the idea of the septate uterus being the most common type among infertile women. ${ }^{19}$ Another study had been performed in Nigeria in which they found that the sub-septate uterus was the predominant type among the infertile population. ${ }^{20}$ Other anomaly types mentioned in our study were also found with lesser frequencies. In this study, a strong association was found between genetic factors and developing Mullerian anomaly. Around $11.5 \%$ abnormal karyotype was detected among the cases, indicating that genetic factor has an important role in the development of such condition. This result agrees with a study conducted in Brazil which found karyotype abnormality in $8 \%$ of cases. $^{21}$ Another study had been performed in Turkey, in which Müllerian agenesis reported in three sisters and their two paternal aunts indicating the importance of the genetic factor in the development of such condition. ${ }^{22}$ However, it is unlikely that a specific gene abnormality is responsible for a specific type of anomaly as different family members can have different types of malformations in their reproductive system and phenotype expression. The success rate of achieving pregnancy after the intervention of each type accordingly was good $(61.1 \%)$, with sub-septate uterus having the highest success rate followed by partial bicornuate and complete septate. Indicating that most types of reproductive anomalies are curable after proper management with either metroplasty, hysteroscopic resection of the septum, and using assisted reproductive technology. In a recent study, a significant reduction of abortion rate found to be from $92 \%$ to $21.2 \%$, and the term delivery rate increased from $0 \%$ to $69.7 \% .{ }^{23}$ This is the first study that had been conducted in the infertility center of Maternity Teaching Hospital in Erbil city to determine the prevalence and pattern of congenital malformations among infertile women. The study has limitations as based on data collection retrospectively from patient files, as many cases were excluded due to incomplete recording of information, missing data, no computerized data about patients. The study evaluates the condition among the infertile population only. For a better understanding of the significance of such problems as a causative factor of infertility, it should be compared with the normal population.

\section{Conclusion}

Congenital anomaly of the female genital tract is not uncommon among infertile women. The sub-septate uterus is the most common type among them; however, the commonness of types may differ in the normal population. The genetic factor has a significant role in the development of such a condition. There is a good prognosis in most types for achieving a successful pregnancy if they managed properly. Sub-septate, incomplete bicornuate, and complete septate having the best success rates, respectively.

\section{Competing interests}

The authors declare no competing interests.

\section{References}

1. Hassan MA, Lavery SA, Trew GH. Congenital uterine anomalies and their impact on fertility. Women's Health 2010; 6(3):443-61.

2. Iverson RE, DeCherney AH, Laufer MR. Clinical manifestations and diagnosis of congenital anomalies of the uterus. Up-To-Date 2012; 20(3):1-4. 
3. Keith L, Moore KL. The developing human: clinically oriented embryology. $10^{\text {th }} \mathrm{ed}$. Philadelphia, PA: Saunders; 2016. P. 51-267.

4. Sadler TW. Urogenital system, Langman's Medical Embryology. $13^{\text {th }}$ ed. Philadelphia, PA: Lippincott Williams \& Wilkins; 2015. P. 261-76.

5. DeSilva M, Munoz FM, Mcmillan M, Kawai AT, Marshall $\mathrm{H}$, Macartney $\mathrm{KK}$, et al. Congenital anomalies: Case definition and guidelines for data collection, analysis, and presentation of immunization safety data. Vaccine 2016; 34(49):6015.

6. Toufaily MH, Westgate MN, Lin AE, Holmes LB. Causes of congenital malformations. Birth Defects Res 2018; 110(2):87-91.

7. Grimbizis GF, Gordts S, Di Spiezio, Sardo A, Brucker S, De Angelis C, et al. The ESHRE/ ESGE consensus on the classification of female genital tract congenital anomalies. Hum Reprod Open 2013; 28(8):44-2032.

8. Acién $P$, Acién Ml. The history of female genital tract malformation classifications and proposal of an updated system. Hum Reprod Update 2011; 17(5):693-705.

9. Heinonen PK. Renal tract malformations associated with Müllerian duct anomalies. Clin Obstet Gynecol Reprod Med 2018; 4(1):3-5.

10. Arbuckle JL, Hoover $\mathrm{KH}$. Development of the Female Reproductive Tract and Associated Anomalies. Curr Treat Options Pediatr 2016; 2(3):131-42.

11. Hua M, Odibo AO, Longman RE, Macones GA, Roehl KA, Cahill AG. Congenital uterine anomalies and adverse pregnancy outcomes. Am J Clin Exp Obstet Gynecol 2011; 205(6): 558.e1-5.

12. Woelfer B, Salim R, Banerjee S, Elson J, Regan L, Jurkovic D. Reproductive outcomes in women with congenital uterine anomalies detected by three-dimensional ultrasound screening. Obstet Gynecol 2001; 98(6):1099-103.

13. Acién $P$, Acién MI. The history of female genital tract malformation classifications and proposal of an updated system. Hum Reprod Update 2011; 17(5):693-705.

14. Grimbizis GF, Gordts S, Di SpiezioSardo A, Brucker S, De Angelis C, Gergolet M, et al. The ESHRE/ESGE consensus on the classification of female genital tract congenital anomalies. Hum Reprod 2013; 28(8):2032-44.

15. Oyetunji I, Aikhuele $\mathrm{H}$, Adejuwon O, Afolabi BM. Pattern of Congenital Uterine Anomalies among Infertile Women With and Without Recurrent Miscarriages, in Southwest Nigeria. Crit Care Obst Gyne 2015; 1(1):7.

16. Robbins JB, Broadwell C, Chow LC, Parry JP, Sadowski EA. Müllerian duct anomalies: embryological development, classification, and MRI assessment. J Magn Reson Imaging 2015; 41(1):1-2.
17. Al-Allaf LI. Frequency of Congenital Anatomical Uterine Abnormalities in a Group of Infertile Women in Mosul city. IJCM 2009; 22(4):238-46.

18. Chan $Y Y$, Jayaprakasan $K$, Zamora J, Thornton JG, Raine-Fenning N, Coomarasamy A. The prevalence of congenital uterine anomalies in unselected and high-risk populations: a systematic review. Hum Reprod Update 2011; 17(6):761-71.

19. Saravelos SH, Cocksedge KA, Li TC. Prevalence and diagnosis of congenital uterine anomalies in women with reproductive failure: a critical appraisal. Hum Reprod Update 2008; 14(5):41529.

20. Ajayi A, Ajayi V, Biobaku O, Oyetunji I, Aikhuele $\mathrm{H}$, Adejuwon $\mathrm{O}$, et al. pattern of congenital uterine anomalies among infertile women with and without recurrent miscarriages, in Southwest Nigeria. Critical Care 2015; 1(1):7.

21. Ribeiro SC, Tormena RA, Peterson TV, Gonzáles MD, Serrano PG, Almeida JA, Baracat EC. Müllerian duct anomalies: review of current management. Sao Paulo Medical Journal 2009; 127(2):92-6.

22. Tiker F, Yildirim SV, Barutcu O, Bağiş T. Familial müllerian agenesis. The Turkish journal of Pediatrics 2000; 42(4):322-4.

23. Tofoski G, Georgievska J. Reproductive Outcome after Hysteroscopic Metroplasty in Patients with Infertility and Recurrent Pregnancy Loss. Macedonian Journal of Medical Sciences 2014; 7(1):8-103. 